\title{
Self-Similar Symmetry Model and Cosmic Microwave Background
}

\author{
Tomohide Sonoda* \\ Graduate School of Natural Sciences, International Christian University, Tokyo, Japan
}

In this paper, we present the self-similar symmetry (SSS) model that describes the hierarchical structure of the universe. The model is based on the concept of self-similarity, which explains the symmetry of the cosmic microwave background (CMB). The approximate length and time scales of the six hierarchies of the universe-grand unification, electroweak unification, the atom, the pulsar, the solar system, and the galactic system - are derived from the SSS model. In addition, the model implies that the electron mass and gravitational constant could vary with the $\mathrm{CMB}$ radiation temperature.

Keywords: self-similarity, symmetry, cosmic microwave background, large numbers hypothesis, large numbers coincidences

\section{OPEN ACCESS}

Edited by:

Peng Gao,

Harvard University, USA

Reviewed by:

Veselin Filev,

Dublin Institute for Advanced Studies,

Ireland

Kazuharu Bamba,

Fukushima University, Japan

${ }^{*}$ Correspondence:

Tomohide Sonoda

tomo@alm.icu.ac.jp

Specialty section:

This article was submitted to

Mathematical Physics,

a section of the journal

Frontiers in Applied Mathematics and

Statistics

Received: 01 January 2016

Accepted: 26 April 2016

Published: 11 May 2016

Citation:

Sonoda T (2016) Self-Similar

Symmetry Model and Cosmic

Microwave Background.

Front. Appl. Math. Stat. 2:5.

doi: 10.3389/fams.2016.00005

\section{INTRODUCTION}

What determines the values of the physical constants and whether they will remain constant over time are fundamental questions in physics. A long-standing conundrum associated with the physical constants is that large dimensionless numbers that are seemingly unrelated can be linked by a scale factor of $10^{39}$ [1-5]. The Dirac large numbers hypothesis (LNH) tackles this problem. It claims that the gravitational constant $G$ is inversely proportional to the age of the universe. The LNH is based on the coincidences between three very large dimensionless numbers $N_{1} \simeq N_{2} \simeq \sqrt{N}$. $N_{1}$, the ratio of the radius of the observable universe to the radius of the electron, is approximately $10^{39} ; N_{2}$, the ratio of the electromagnetic and gravitational forces between a proton and an electron, is also approximately $10^{39}$; and $\mathrm{N}$, the number of protons in the observable universe, is approximately $10^{78}$. The Dirac LNH argues that "any two of the very large dimensionless numbers occurring in Nature are connected by a simple mathematical relation, in which the coefficients are of the order of unity"[1].

In this paper, we present the self-similar symmetry (SSS) model in which the relationships among these seemingly unrelated physical quantities are represented using a simple geometric sequence for which the first term and the geometric ratio are given by dimensionless ratios of masses. Based on the LNH, the first term of the geometric sequence corresponds to the cosmic microwave background $(\mathrm{CMB})$ radiation temperature, which points to the possibility that the values of the physical constants are determined by the $\mathrm{CMB}$ radiation temperature.

\section{THE SELF-SIMILAR SYMMETRY MODEL}

In the SSS model, the CMB has a symmetrical self-similar structure and the physical constants are dimensionless, otherwise they would not have universality. Therefore, the fundamental dimensionless mass ratios are defined as follows:

$$
A=\log \alpha=\log \left(\frac{m_{\mathrm{pl}}}{m_{\mathrm{pr}}}\right), B=\log \beta=\log \left(\frac{m_{\mathrm{e}}}{m_{\mathrm{pr}}}\right),
$$


where $m_{\mathrm{pr}}$ is the proton mass, $m_{\mathrm{e}}$ is the electron mass, and $m_{\mathrm{pl}}$ is the Planck mass, and the fundamental dimensionless time and length ratios are defined as

$$
T=\log \left(\frac{t}{t_{\mathrm{pl}}}\right), L=\log \left(\frac{l}{l_{\mathrm{pl}}}\right),
$$

where $t$ and $l$ are the time and length scales of the hierarchies and $t_{\mathrm{pl}}$ and $l_{\mathrm{pl}}$ are the Planck time and Planck length, respectively. The similarity dimension $D$ is defined using these dimensionless parameters:

$$
D=\left(\frac{T}{L}\right)^{3}=\frac{A}{A+B} \approx 1.206 .
$$

We then assume that hierarchical structures are constructed according to the following sequences:

$$
\begin{array}{rlrl}
L_{0} & =2(A+B) \approx 31.701, \\
L_{n} & =D^{n} L_{0} & \text { for } \quad L>L_{0}, \\
L_{m} & =\left(2-D^{m}\right) L_{0} & \text { for } \quad L<L_{0},
\end{array}
$$

where $n$ and $m$ are natural numbers that represent the hierarchical level. In addition, the time scales of each hierarchy are calculated using Equation (3).

\section{VERIFICATION OF THE SSS MODEL}

To verify the SSS model, we compared values obtained with it against reference values. Tables 1, 2 summarize the length and time scales, respectively, of the grand unification, electroweak unification, atom, pulsar, solar system, and galactic system hierarchies of the universe. The SSS model values agree well with the reference values. Figure 1 shows the hierarchy time scale as a function of the length scale. The coincidences in the figure confirm the validity of the SSS model.

TABLE 1 | Length scales of the hierarchies of the universe.

\begin{tabular}{lcccc}
\hline \multicolumn{1}{c}{ Hierarchy } & $\boldsymbol{I}(\mathbf{m})$ & $\boldsymbol{L}$ & Sss model & Error $(\%)$ \\
\hline Planck $^{\mathrm{a}}$ & $1.6 \times 10^{-35}$ & 0 & - & \multicolumn{1}{c}{-} \\
Grand unification $^{\mathrm{b}}$ & $10^{-27}$ & 7.79 & $7.81(\mathrm{~m}=3)$ & 0.2 \\
Electroweak unification $^{\mathrm{b}}$ & $10^{-17}$ & 17.79 & $17.30(\mathrm{~m}=2)$ & -2.7 \\
Atom $^{\mathrm{c}}$ & $2.4 \times 10^{-10}$ & 25.17 & $25.17(\mathrm{~m}=1)$ & 0.0 \\
Pulsar $^{\mathrm{d}}$ & $2.4 \times 10^{4}$ & 39.17 & $38.23(n=1)$ & -2.4 \\
Solar system $^{\mathrm{e}}$ & $3.0 \times 10^{11}$ & 46.27 & $46.10(n=2)$ & -0.3 \\
Galaxy $^{f}$ & $5.3 \times 10^{20}$ & 55.52 & $55.59(n=3)$ & 0.1 \\
\hline
\end{tabular}

${ }^{a}$ The Planck length $I_{p l}$ is defined as $I_{p l}=\sqrt{\hbar G / C^{3}}$, where $\hbar$ is the Dirac's constant, $G$ is the gravitational constant, and $c$ is the speed of light in a vacuum.

${ }^{b}$ Values are taken from a magnetic monopole structure in grand unified theories (GUTS) [2].

${ }^{c}$ Assumed to be twice the van der Waals radius of a hydrogen atom [6].

${ }^{d}$ Estimated to be 1.5 times the mass of the Sun with a radius of $12 \mathrm{~km}$ [7].

e Based on the average diameter of the Earth's orbit around the Sun; 2 astronomical units (AUs) [6].

${ }^{f}$ Taken as twice the distance from the center of the galaxy to the solar system, which is 28,000 light years [6].

\section{DISCUSSION}

From Equation (1), $2 A=-\log \alpha_{\mathrm{G}}$, where $\alpha_{\mathrm{G}}=G m_{\mathrm{pr}}^{2} / \hbar c$ is the gravitational coupling constant, the following coincidences occurs:

$$
L_{n=2}-L_{m=3}=L_{n=3}-L_{m=2} \approx 2 A \text {. }
$$

Equation (7) shows that $\alpha_{\mathrm{G}}$ plays an important role in forming the hierarchical structure of the universe. In addition,

$$
L_{m=1}-L_{0}=L_{0}-L_{n=1}=2 B
$$

\section{TABLE 2 | Time Scales of the Hierarchies of the Universe ${ }^{a}$.}

\begin{tabular}{lcccc}
\hline \multicolumn{1}{c}{ Hierarchy } & $\boldsymbol{t}(\mathbf{s})$ & $\boldsymbol{T}$ & SSS model & Error (\%) \\
\hline Planck $^{\mathrm{b}}$ & $5.4 \times 10^{-44}$ & 0 & - & - \\
Grand unification $^{\mathrm{c}}$ & $2.2 \times 10^{-35}$ & 8.61 & $8.31(m=3)$ & -3.5 \\
Electroweak unification $^{\mathrm{d}}$ & $6.6 \times 10^{-27}$ & 17.09 & $18.42(m=2)$ & 7.7 \\
Atom $^{\mathrm{C}}$ & $4.8 \times 10^{-17}$ & 26.95 & $26.79(m=1)$ & -0.6 \\
Pulsar $^{f}$ & $2.9 \times 10^{-2}$ & 41.72 & $40.69(n=1)$ & -2.5 \\
Solar system $^{\mathrm{y}}$ & $3.2 \times 10^{7}$ & 50.77 & $49.07(n=2)$ & -3.3 \\
Galaxy $^{\text {h }}$ & $7.6 \times 10^{15}$ & 59.15 & $59.17(n=3)$ & 0.0 \\
\hline
\end{tabular}

a The orbital motion and magnetic fields of the Earth and Sun cause emitted light to have a long period. Therefore, although the physical structures are different in each hierarchy, their time scales can be compared in a unifying manner using the period of light.

${ }^{b}$ Planck time $t_{p l}=\sqrt{\hbar G / c^{5}}$.

${ }^{c}$ Grand unification at the intermediate mass scale $M_{\text {GUT }}^{\prime} \approx 3 \times 10^{10} \mathrm{GeV}$ proposed by Dienes et al. [8]; $t=\hbar /\left(3 \times 10^{19}\right) \mathrm{s}$.

${ }^{d}$ Electromagnetic force and weak force unify at $10^{2} \mathrm{GeV}[9] ; t=\hbar / 10^{11} \mathrm{~s}$.

${ }^{e}$ Based on the first ionization energy of hydrogen [6]; $t=\hbar / 13.6 \mathrm{~s}$.

${ }^{f}$ Inverse of the average observed frequency of $f_{\text {pulsar }} \approx 35 \mathrm{~Hz}(N=2,307$ pulsars $)$ determined from [10].

${ }^{g}$ Period of the Earth's revolution around the Sun [6].

${ }^{h}$ The revolution of the Sun around the center of the Milky Way, i.e., 1 galactic year $\approx 240$ million years, based on a galactic rotational speed of approximately $220 \mathrm{~km} / \mathrm{s}$ [6].

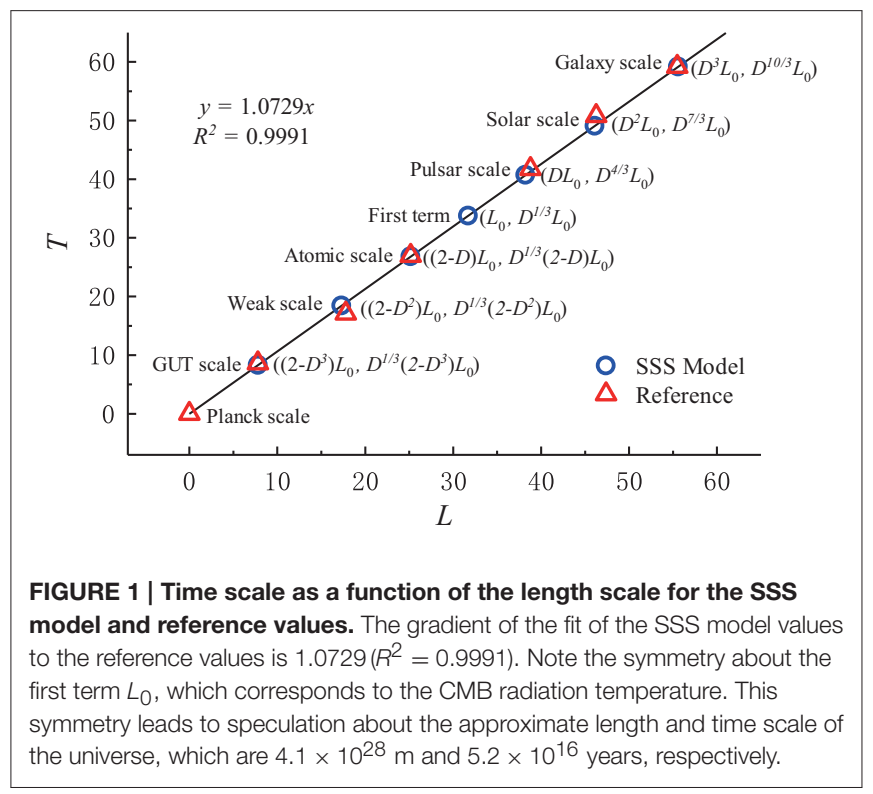


Thus, if $L_{\mathrm{u}}$ is the length of the universe, the following hierarchy holds:

$$
L_{\mathrm{u}}=2 L_{0}
$$

Therefore, the ratios of the coincidences between the length scales of the hierarchies are

$$
\begin{aligned}
& r_{a}=\frac{L_{n=2}-L_{m=3}}{L_{n=1}} \approx 1.002, \\
& r_{b}=\frac{L_{\mathrm{u}}-L_{n=3}}{L_{n=2}-L_{n=1}} \approx 0.991,
\end{aligned}
$$

from which we get

$$
D=\frac{r_{a}-r_{b}}{1-r_{b}}
$$

From Equation (12), we see that $r_{a} \neq r_{b} \neq 1$.

With respect to the first term of the geometric sequence, $L_{0}$, we find that

$$
(\alpha \beta)^{-2} T_{\mathrm{pl}} \approx 2.821 \mathrm{~K},
$$

where $T_{\mathrm{pl}}$ is the Planck temperature. The value in Equation (13) is consistent with the $\mathrm{CMB}$ radiation temperature $T_{\mathrm{CMB}}[11]$. Assuming the right-hand side of Equation (13) represents the $T_{\mathrm{CMB}}$, if $\mathrm{LNH}$ is applied to Equation (13) and we define the dimensionless temperature ratio $\tau_{\mathrm{CMB}}=T_{\mathrm{CMB}} / T_{\mathrm{pl}}$, we get

$$
\alpha_{\mathrm{G}} \approx \tau_{\mathrm{CMB}}^{D}
$$

Similarly,

$$
\beta^{2} \approx \tau_{\mathrm{CMB}}^{D-1}
$$

\section{REFERENCES}

1. Dirac PAM. The cosmological constants. Nature (1937) 139:323. doi: 10.1038/139323a0

2. Barrow JD, Tipler FJ. The Anthropic Cosmological Principle. New York, NY: Oxford University Press (1986). p. 656.

3. Dicke RH. Dirac's cosmology and Mach's principle. Nature (1961) 192:440-1. doi: 10.1038/192440a0

4. Carter B. In: Longair MS, editor. Confrontation of Cosmological Theories with Observational Data. Dordrecht: Reidel (1974), p. 291-8. doi: 10.1007/978-94010-2220-0_25

5. Carr BJ, Rees MJ. The anthropic principle and the structure of the physical world. Nature (1979) 278:605-12. doi: 10.1038/278605a0

6. National Astronomical Observatory of Japan. Chronological Scientific Tables [in Japanese]. Tokyo: Maruzen Publishing Co. (2013).

7. Lattimer JM Prakash M. The physics of neutron stars. Science (2004) 304:53642. doi: 10.1126/science. 1090720

8. Dienes KR, Dudas E, Gherghetta T. Grand unification at intermediate mass scales through extra dimensions. Nucl Phys B (1999) 537:47-108. doi: 10.1016/S0550-3213(98)00669-5
Thus, both $\alpha_{\mathrm{G}}$ and $\beta$ are power functions of $T_{\mathrm{CMB}}$.

Substituting $T_{\mathrm{CMB}}=T_{\mathrm{pl}}$, an initial condition of the universe, into Equations (14) and (15) yields $\alpha=\beta=1$, which means that the entire hierarchy was contained in a single point and that the electron, proton, and Planck masses were equivalent. These masses have varied since that initial single point such that $m_{\mathrm{e}} \ll m_{\mathrm{pr}} \ll m_{\mathrm{pl}}$, in response to the changing $T_{\mathrm{CMB}}$, where $T_{\mathrm{CMB}} \ll T_{\mathrm{pl}}$. Assuming that $T_{\mathrm{CMB}} \rightarrow 0$ is the ultimate fate of the universe, then $\alpha \rightarrow \infty, \beta \rightarrow 0$, and $L_{0} \rightarrow \infty$, indicating that $m_{\mathrm{e}} \rightarrow 0$ and $G \rightarrow 0$ as the universe expands to infinity.

\section{CONCLUSIONS}

Our SSS model describes the large-scale structure of the universe and shows that the six hierarchies of the universe are self-similar to the $\mathrm{CMB}$, indicating that the $\mathrm{CMB}$ is key to unifying quantum theory with general relativity. In addition, the SSS model leads to the conclusion that $m_{\mathrm{e}}$ and $G$ vary with $T_{\mathrm{CMB}}$. Any errors arising from the SSS model are problems to be tackled in the future.

\section{AUTHOR CONTRIBUTIONS}

TS conceived the study and prepared the manuscript.

\section{ACKNOWLEDGMENTS}

The author thanks M. B. Greenfield and K. Kitahara for helpful discussions.

9. Bertone G, Hooper D, Silk J. Particle dark matter: evidence, candidates and constraints. Phys Rep. (2005) 405:279-390. doi: 10.1016/j.physrep.2004.0 8.031

10. Manchester RN, Hobbs GB, Teoh A, Hobb M. The Australia telescope national facility pulsar catalogue. Astron J. (2005) 129:1993-2006. doi: $10.1086 / 428488$

11. Fixsen DJ. The temperature of the cosmic microwave background. Astrophys J. (2009) 707:916-20. doi: 10.1088/0004-637X/707/ 2/916

Conflict of Interest Statement: The author declares that the research was conducted in the absence of any commercial or financial relationships that could be construed as a potential conflict of interest.

Copyright (C) 2016 Sonoda. This is an open-access article distributed under the terms of the Creative Commons Attribution License (CC BY). The use, distribution or reproduction in other forums is permitted, provided the original author(s) or licensor are credited and that the original publication in this journal is cited, in accordance with accepted academic practice. No use, distribution or reproduction is permitted which does not comply with these terms. 